\title{
COVID-19 Vaccination Hesitancy in the United States: A Rapid National Assessment
}

\author{
Jagdish Khubchandani ${ }^{1}$. Sushil Sharma ${ }^{2}$. James H. Price ${ }^{3} \cdot$ Michael J. Wiblishauser $^{4} \cdot$ Manoj Sharma $^{5} \cdot$ Fern J. Webb $^{6}$
}

Accepted: 10 December 2020 / Published online: 3 January 2021

(c) The Author(s), under exclusive licence to Springer Science+Business Media, LLC part of Springer Nature 2021

\begin{abstract}
Given the results from early trials, COVID-19 vaccines will be available by 2021 . However, little is known about what Americans think of getting immunized with a COVID-19 vaccine. Thus, the purpose of this study was to conduct a comprehensive and systematic national assessment of COVID-19 vaccine hesitancy in a community-based sample of the American adult population. A multi-item valid and reliable questionnaire was deployed online via mTurk and social media sites to recruit U.S. adults from the general population. A total of 1878 individuals participated in the study where the majority were: females (52\%), Whites (74\%), non-Hispanic (81\%), married (56\%), employed full time (68\%), and with a bachelor's degree or higher (77\%). The likelihood of getting a COVID-19 immunization in the study population was: very likely (52\%), somewhat likely $(27 \%)$, not likely (15\%), definitely not (7\%), with individuals who had lower education, income, or perceived threat of getting infected being more likely to report that they were not likely/definitely not going to get COVID-19 vaccine (i.e., vaccine hesitancy). In unadjusted group comparisons, compared to their counterparts, vaccine hesitancy was higher among African-Americans (34\%), Hispanics (29\%), those who had children at home (25\%), rural dwellers (29\%), people in the northeastern U.S. (25\%), and those who identified as Republicans (29\%). In multiple regression analyses, vaccine hesitancy was predicted significantly by sex, education, employment, income, having children at home, political affiliation, and the perceived threat of getting infected with COVID-19 in the next 1 year. Given the high prevalence of COVID-19 vaccine hesitancy, evidence-based communication, mass media strategies, and policy measures will have to be implemented across the U.S. to convert vaccines into vaccinations and mass immunization with special attention to the groups identified in this study.
\end{abstract}

Keywords COVID-19 $\cdot$ Pandemic $\cdot$ Vaccine $\cdot$ Public health $\cdot$ Prevention $\cdot$ Infection

Jagdish Khubchandani

jagdish@nmsu.edu

1 Department of Public Health Sciences, New Mexico State University, Las Cruces, NM 88003, USA

2 Miller College of Business, Ball State University, Muncie, IN 47306, USA

3 Department of Population Health, Emeritus Professor of Public Health, University of Toledo, Toledo, $\mathrm{OH} 43606$, USA

4 Department of Health Studies, University of Houston-Victoria, Victoria, TX 77901, USA

5 Department of Environmental \& Occupational Health, University of Nevada, Las Vegas, NV 89119, USA

6 Department of Community Health and Family Medicine, University of Florida, Jacksonville, FL 32209, USA

\section{Background}

The community, economic, and health tolls of COVID-19 in the United States (U.S) have been among the highest in the world. By November 2020, more than 10 million people had been infected and more than a quarter-million died of COVID-19 in the U.S. [1, 2] However, by late November, several pharmaceutical companies announced the early results of their large vaccine trials claiming efficacy for the majority of the trial participants [3]. Federal and regulatory approvals were being sought by pharmaceutical companies in early December and various state and national agencies started discussing the acquisition, storage, and deployment of vaccines [3-5]. Simultaneously, popular media brought to attention a constant problem plaguing U.S population health in recent years, vaccine hesitancy. Antivax groups' statements, conspiracy theories, myths and misperceptions, questions about the speed of vaccine development and 
long-term side effects, and expert opinion on challenges with the COVID-19 vaccine were proliferating in the national media. The scientific community and public health experts expressed concerns as early as summer 2020 about vaccine hesitancy in the U.S, in addition to the challenges associated with costs, access, effectiveness, and logistics of vaccine deployment [6-12]. Vaccine hesitancy is not a novel phenomenon in the U.S, the most recent example being of the reemergence of measles in the years preceding the COVID-19 pandemic [10-12]. Similarly, there are population subgroups around the world with high vaccine hesitancy reported before the pandemic. In fact, before the COVID-19 pandemic, the World Health Organization mentioned vaccine hesitancy as one of the top global health threats [9-12]. While there are considerable enthusiasm and anticipation for the COVID-19 vaccine, little is known about vaccine hesitancy specifically for COVID-19 in the U.S population. Identifying specific populations and their characteristics with regards to vaccine hesitancy will help serve as key components of a successful vaccination strategy when a COVID-19 vaccine is available for the general population. Thus, this study aimed to conduct a comprehensive national assessment of COVID-19 related vaccine hesitancy in the U.S and to identify population subgroups with a higher probability of vaccine hesitancy.

\section{Methods and Procedures}

A multi-item questionnaire was created based on a comprehensive literature review (to ensure face validity) and based on expert suggestions (to ensure content validity). The survey was deployed using Amazon mTurk in June 2020. The survey was also shared and posted on various social media sites (e.g. Facebook and Twitter) and networks such as academic posts, community organizations, and in personal groups. The questionnaire could be taken only once on computers and mobile phones with all anonymity and privacy conditions for data provided upfront to potential study participants. To estimate the required sample size, an a priori power analysis was conducted. Based on the total population of adults in the US ( $\mathrm{n}=\approx 250$ million), with $99 \%$ confidence levels, and a conservative 3\% margin of error, a total of 1383 participants were needed for the study. The study protocol and procedures were approved by the Institutional Review Board before the survey was distributed [13, 14].

The first question on the survey asked about the likelihood of getting a vaccine: "If a vaccine was available that would prevent coronavirus infection, how likely is it that you would get the vaccine/shot" The response options were: very likely, somewhat likely, not likely, definitely not. This measure served as the major dependent variable and a key outcome of the study (i.e., vaccine hesitancy). Next, we asked two questions to assess the perceived COVID-19 threat. First, "How likely is it that you or a family member could get infected with coronavirus in the next 1 year" with response options: very likely, somewhat likely, not likely, and definitely not. Second, "How concerned are you that you or a family member could get infected with coronavirus in the next 1 year" with response options: very concerned, concerned, slightly concerned, and not concerned at all. A final set of closed format questions with predetermined options assessed participants' sex, age, race, ethnicity, marital status, education, employment status, household income, the region of residence in the US, area characteristics of residence, political affiliation, and whether or not the participants had children at home.

Data were analyzed using SPSS 22 (IBM Corp). We computed descriptive statistics to describe the demographic characteristics of the study participants. Next, vaccine hesitancy proportions were computed and compared across groups using Chi square tests. Responses were compared for various sociodemographic characteristics by dichotomizing the variable as a positive (very likely and somewhat likely) or negative attitude (not likely and definitely not) towards a COVID-19 vaccine indicating the extent of vaccine hesitancy. Multiple logistic regression analyses were conducted with vaccine hesitancy as an outcome and perceived COVID-19 threat and sociodemographic characteristics as predictor variables to compute the adjusted odds ratios (AOR) with 95\% confidence intervals for vaccine hesitancy. Hosmer-Lemeshow goodness-of-fit tests were used to ensure that models adequately fit the data. Statistical significance was established at an alpha of $p<0.05$.

\section{Results}

A total of 1878 adults participated in this study. The majority of the study participants were: females (52\%), Whites (74\%), non-Hispanic (81\%), married (56\%), employed full time $(68 \%)$ and had a bachelor's degree or higher $(77 \%)$ (Table 1). Regarding the question on the likelihood of getting a COVID-19 vaccine, the responses were: very likely (52\%), somewhat likely (27\%), not likely (15\%), definitely not (7\%). Statistically significant differences in vaccine hesitancy were found based on sociodemographic characteristics with the highest prevalence of COVID-19 vaccine hesitancy in African-Americans, Hispanics, those who had children at home, individuals with lower education and incomes, rural dwellers, people in the northeastern US, and those who identified as Republicans (Table 1).

Also, those who were less likely to believe that they or a family member could be infected with COVID-19 in the next 1 year and those who were least concerned about themselves or a family member getting COVID-19 infection in the next 
Table 1 Sociodemographic characteristics, COVID-19 threat, and vaccine hesitancy

\begin{tabular}{|c|c|c|c|c|}
\hline \multirow[t]{2}{*}{ Variable } & \multirow{2}{*}{$\begin{array}{l}\text { Total sample } \\
\mathrm{n}(\%)\end{array}$} & \multicolumn{3}{|c|}{ Likelihood of getting COVID vaccine } \\
\hline & & $\begin{array}{l}\text { Very likely/some- } \\
\text { what likely n (\%) }\end{array}$ & $\begin{array}{l}\text { Not likely/defi- } \\
\text { nitely not n (\%) }\end{array}$ & $\mathrm{p}$ value \\
\hline All participants & $1878(100)$ & $1467(78)$ & $411(22)$ & - \\
\hline Sex & & & & 0.81 \\
\hline Male & $910(48)$ & $709(78)$ & $201(22)$ & \\
\hline Female & $968(52)$ & $758(78)$ & $210(22)$ & \\
\hline Age group & & & & 0.06 \\
\hline $18-25$ years & $349(19)$ & $271(78)$ & $78(22)$ & \\
\hline $26-40$ years & $829(44)$ & $645(78)$ & $184(22)$ & \\
\hline $41-60$ years & $525(28)$ & $401(76)$ & $124(24)$ & \\
\hline$\geq 61$ years & $175(9)$ & $150(86)$ & $25(14)$ & \\
\hline Race & & & & $<0.001$ \\
\hline White & $1384(74)$ & $1083(78)$ & $301(22)$ & \\
\hline Black & $214(11)$ & $142(66)$ & $72(34)$ & \\
\hline Asian & $173(9)$ & $159(89)$ & $20(11)$ & \\
\hline Multiracial & $43(2)$ & $36(84)$ & $7(16)$ & \\
\hline Other & $58(3)$ & $47(81)$ & $11(19)$ & \\
\hline Ethnicity & & & & 0.001 \\
\hline Hispanic & $357(19)$ & $255(71)$ & $102(29)$ & \\
\hline Non-Hispanic & $1521(81)$ & $1212(80)$ & $309(20)$ & \\
\hline Marital status & & & & 0.12 \\
\hline Single/never married & $611(33)$ & $487(80)$ & $124(30)$ & \\
\hline Married & $1050(56)$ & $810(77)$ & $240(23)$ & \\
\hline Engaged/living with a partner & $98(5)$ & $83(85)$ & $15(15)$ & \\
\hline Divorced/separated/widow & $119(6)$ & $87(74)$ & $32(16)$ & \\
\hline Children at home & & & & 0.001 \\
\hline Yes & $882(47)$ & $657(75)$ & $225(25)$ & \\
\hline No & $996(53)$ & $810(81)$ & $186(19)$ & \\
\hline Education & & & & $<0.001$ \\
\hline$\leq$ High school & $104(6)$ & $72(69)$ & $32(31)$ & \\
\hline Some college education & $315(17)$ & $234(74)$ & $81(26)$ & \\
\hline Bachelor's degree & $910(48)$ & $686(75)$ & $224(25)$ & \\
\hline$\geq$ Master's degree & $549(29)$ & $475(87)$ & $74(13)$ & \\
\hline Current employment status & & & & 0.07 \\
\hline Full-time & $1274(68)$ & $982(77)$ & $292(23)$ & \\
\hline Part-time & $302(16)$ & $234(78)$ & $168(22)$ & \\
\hline Not employed & $302(16)$ & $251(83)$ & $51(17)$ & \\
\hline Annual household income & & & & $<0.001$ \\
\hline $0-\$ 30,000$ & $337(18)$ & $241(72)$ & $96(28)$ & \\
\hline$\$ 30,001-60,000$ & $610(33)$ & $454(74)$ & $156(26)$ & \\
\hline$\$ 60,001-99,999$ & $533(28)$ & $429(81)$ & $104(19)$ & \\
\hline$\geq \$ 100,000$ & $390(21)$ & $343(86)$ & $55(14)$ & \\
\hline Area of residence & & & & $<0.001$ \\
\hline Rural & $409(22)$ & $292(71)$ & $117(29)$ & \\
\hline Urban & $765(40)$ & $599(78)$ & $166(22)$ & \\
\hline Suburban & $704(38)$ & $576(82)$ & $128(18)$ & \\
\hline Region in USA & & & & 0.02 \\
\hline Northeast & $244(13)$ & $184(75)$ & $60(25)$ & \\
\hline Midwest & $639(34)$ & $525(82)$ & $114(18)$ & \\
\hline South & $566(30)$ & $434(77)$ & $132(23)$ & \\
\hline West & $429(23)$ & $324(76)$ & $105(24)$ & \\
\hline
\end{tabular}


Table 1 (continued)

\begin{tabular}{|c|c|c|c|c|}
\hline \multirow[t]{2}{*}{ Variable } & \multirow{2}{*}{$\begin{array}{l}\text { Total sample } \\
\mathrm{n}(\%)\end{array}$} & \multicolumn{3}{|c|}{ Likelihood of getting COVID vaccine } \\
\hline & & $\begin{array}{l}\text { Very likely/some- } \\
\text { what likely n (\%) }\end{array}$ & $\begin{array}{l}\text { Not likely/defi- } \\
\text { nitely not } \mathrm{n}(\%)\end{array}$ & $\mathrm{p}$ value \\
\hline \multicolumn{4}{|l|}{ Political orientation } & $<0.001$ \\
\hline Democrat & $863(46)$ & $721(84)$ & $142(16)$ & \\
\hline Republican & $513(28)$ & $365(71)$ & $148(29)$ & \\
\hline Independent & $364(19)$ & $274(75)$ & $90(25)$ & \\
\hline Other & $138(7)$ & $107(78)$ & $31(22)$ & \\
\hline \multicolumn{4}{|c|}{ Perceived likelihood of getting infected in the next 1 year } & $<0.001$ \\
\hline Very likely & 349 (19) & $314(90)$ & $35(10)$ & \\
\hline Somewhat likely & $925(49)$ & $751(81)$ & $174(19)$ & \\
\hline Not likely & $479(25)$ & $349(73)$ & $130(27)$ & \\
\hline Definitely not & $125(7)$ & $53(42)$ & $72(58)$ & \\
\hline \multicolumn{4}{|c|}{ Level of concern about getting infected in the next 1 year } & $<0.001$ \\
\hline Very concerned & $490(26)$ & $425(87)$ & $65(13)$ & \\
\hline Concerned & $698(37)$ & $595(85)$ & $103(15)$ & \\
\hline Slightly concerned & $538(29)$ & $375(70)$ & $163(30)$ & \\
\hline Not concerned at all & $152(8)$ & $72(47)$ & $80(53)$ & \\
\hline
\end{tabular}

$\mathrm{N}(\%)$ indicates frequency and percentage of individuals who selected an option on the variables. $\mathrm{p}$ value indicates level of alpha for statistical significance

1 year had the highest rates of COVID-19 vaccine hesitancy (Table 1).

Although key differences were found between groups on vaccine hesitancy, we conducted a multiple regression analysis to examine factors that could predict COVID-19 vaccine hesitancy (Table 2). In this multiple regression model, compared to their counterparts, the groups with statistically significantly higher odds of vaccine hesitancy were: females (AOR $=1.44$ ), those with children at home $(\mathrm{AOR}=1.34)$, Republicans $(\mathrm{AOR}=1.38)$ and Independents $(\mathrm{AOR}=1.44)$, and those who were slightly concerned ( $\mathrm{AOR}=2.21)$ or not concerned at all $(\mathrm{AOR}=3.80)$ about themselves or a family member getting infected with COVID-19 in the next 1 year. Similarly, compared to those who believed it was very likely that they or their family members could be infected with COVID-19 in the next year, those who thought such an occurrence would be somewhat likely $(\mathrm{AOR}=2.00)$, not likely $(\mathrm{AOR}=2.32)$, or definitely not $(\mathrm{AOR}=6.47)$ had significantly higher odds of vaccine hesitancy. In contrast, those who were not working $(\mathrm{AOR}=0.63)$ or had incomes $\geq 60,001$ (AOR $=0.64)$, or had education $\geq$ college degrees $(\mathrm{AOR}=0.63$ ) had statistically significantly lower odds for vaccine hesitancy.

\section{Discussion}

In this large national study of adult Americans, more than a fifth of the participants (22\%) reported COVID-19 vaccine hesitancy. In a very large June 2020 global survey of more than 13,000 adults from more than a dozen countries including the U.S, $71.5 \%$ of participants worldwide reported that they would be very or somewhat likely to take a COVID-19 vaccine. The proportion of U.S-based participants who reported willingness to take a COVID-19 vaccine was $75.2 \%$, lower than participants from many low- and middle-income countries in the same study [15]. Another April 2020 study of 991 American adults found that $57.6 \%$ of participants intended to be vaccinated, $31.6 \%$ were not sure, and $10.8 \%$ did not intend to be vaccinated [16]. A May 2020 study of more than 5000 American adults found that almost a third $(31.1 \%)$ of participants did not intend on getting a COVID-19 vaccine [17]. These differences could be in part due to the timing of studies and data collection and the way questions were asked across studies. For example, a Kaiser Family Foundation (KFF) poll from the last week of August 2020 found that only $42 \%$ of the participants would want to get the COVID-19 vaccine if approved before the U.S presidential elections in November 2020. The reason for such high vaccine hesitancy could be explained in part by another response from the participants in this KFF poll where a majority (62\%) believed that sociopolitical factors and pressures may lead to a rushed approval for the COVID vaccine without the assurances of safety and efficacy [18]. This issue remained of concern even after the first trial results were announced in late November for potential vaccine candidates. The U.S public and scientific experts were expecting more information on vaccine efficacy, safety, long term side effects, and details of results from the trials 
Table 2 Multiple regression analysis-predictors of vaccine hesitancy in study participants

\begin{tabular}{|c|c|c|c|c|}
\hline Variables & $\mathrm{B}$ & Wald & $\mathrm{p}$ value & $\operatorname{AOR}(95 \% \mathrm{CI})$ \\
\hline Sex (female vs. male) & .362 & 8.100 & .004 & $1.44(1.12-1.84)$ \\
\hline Age & -.001 & .065 & .799 & $0.99(0.98-1.01)$ \\
\hline Race & -.089 & 1.763 & .184 & $0.92(0.80-1.05)$ \\
\hline Ethnicity & -.237 & 2.197 & .138 & $0.79(0.58-1.08)$ \\
\hline Marital Status & .087 & .944 & .331 & $1.09(0.92-1.30)$ \\
\hline Children at home (yes vs. no) & .288 & 4.587 & .032 & $1.34(1.03-1.74)$ \\
\hline \multicolumn{5}{|l|}{ Employment } \\
\hline Full time & Ref & 5.824 & - & Ref \\
\hline Part time & -.173 & 1.051 & .305 & $0.84(0.61-1.18)$ \\
\hline Not working & -.458 & 5.577 & .018 & $0.63(0.44-0.93)$ \\
\hline Area of residence & -.109 & 1.706 & .191 & $0.90(0.76-1.06)$ \\
\hline Income $(\geq \$ 60,001$ vs. $\leq 60,000)$ & -.454 & 12.451 & .001 & $0.64(0.49-0.81)$ \\
\hline Region & .002 & .001 & .973 & $1.01(0.89-1.14)$ \\
\hline Education ( $\geq$ college degree vs. $<$ college degree) & -.450 & 9.290 & .002 & $0.63(0.48-0.85)$ \\
\hline \multicolumn{5}{|l|}{ Political orientation } \\
\hline Democrat & Ref & 7.609 & - & Ref \\
\hline Republican & .319 & 4.586 & .032 & $1.38(1.03-1.84)$ \\
\hline Independent & .362 & 4.738 & .030 & $1.44(1.04-2.00)$ \\
\hline Other & .410 & 2.858 & .091 & $1.51(0.94-2.43)$ \\
\hline \multicolumn{5}{|l|}{ Level of concern about getting infected } \\
\hline Very concerned & Ref & 57.357 & - & Ref \\
\hline Concerned & -.030 & .028 & .866 & $.97(0.68-1.38)$ \\
\hline Slightly concerned & .791 & 19.709 & .001 & $2.21(1.56-1.38)$ \\
\hline Not concerned at all & 1.333 & 31.538 & .001 & $3.80(2.39-6.03)$ \\
\hline \multicolumn{5}{|l|}{ Perceived likelihood of getting infected } \\
\hline Very likely & Ref & 45.181 & - & Ref \\
\hline Somewhat likely & 689 & 11.060 & .001 & $2.00(1.33-2.99)$ \\
\hline Not likely & .838 & 13.729 & .000 & $2.32(1.49-3.60)$ \\
\hline Definitely not & 1.867 & 44.306 & .000 & $6.47(3.74-11.21)$ \\
\hline
\end{tabular}

AOR indicates adjusted odds ratio for the likelihood of vaccine hesitancy (i.e. individuals who were not likely or were definitely not going to get COVID-19 vaccine)

95\% CI indicates 95\% confidence intervals for adjusted odds ratios. p value indicates significance levels. Bold indicates statistically significantly higher or lower odds for vaccine hesitancy. Ref indicates reference/ comparison group among all response options for a variable claiming success for a COVID-19 vaccine. Effective communication on safety and efficacy along with greater transparency on vaccine development and distribution including cost aspects should remain a cornerstone for all other strategies to ensure equitable mass immunization programs related to the COVID-19 vaccine [8-10, 15-18].

Racial and ethnic minorities had higher vaccine hesitancy in group comparisons (African-Americans $=34 \%$ and Hispanics $=29 \%$ ). This could be explained based on a variety of factors that existed before the COVID-19 pandemic such as preexisting vaccine hesitancy, lower access and interaction with healthcare professionals for minorities, historical biomedical and healthcare-related mistrust, lower participation of minorities in clinical trials, cost-related concerns, lesser believe in the scientific enterprise of medicine and public health, lower awareness, and education [19-22]. For example, Callaghan and colleagues reported in their study that African-Americans were more hesitant on COVID-19 vaccines due to concerns about safety and efficacy, they lack needed financial resources or health insurance, or they already had COVID-19. [17]. Unfortunately, racial/ethnic minorities also have the worst outcomes associated with COVID-19 infection [17, 20]. Culturally competent strategies for public health practice and research have shown promise in improving health outcomes and engagement in preventive behaviors of minorities and should be considered when planning the deployment of COVID-19 vaccines [17, 19-21].

Individuals living in rural areas, those with lower household incomes, and lower levels of education were more likely to be hesitant about getting immunized with a COVID-19 vaccine. Studies by Callaghan et al. and Fisher 
et al. also reported that individuals with lower income and education and those who lived in rural America were less likely to pursue COVID-19 vaccination [16, 17]. COVID19 vaccine hesitancy in rural, lower-income, low education groups can be explained by preexisting vaccine hesitancy in these groups, lower awareness and health literacy, lower trust and interaction with healthcare professionals, and costbased concerns [17, 19-22]. A unique finding of this study that could also relate to income and education is vaccine hesitancy based on employment status with the unemployed $(\mathrm{AOR}=0.63)$ having the lowest odds for vaccine hesitancy. The Israeli study by Dror and colleagues also found that those who lost a job or were not working were significantly more likely to accept a COVID-19 vaccine than those who continued to work during the pandemic [7]. It could be possible that those who are not working would like to return to work and employment and the vaccine could be a factor that could facilitate return to work. However, these observations are in contrast to the findings of Malik and colleagues who reported that the employed were more likely to accept a COVID-19 vaccine than unemployed study participants in the U.S. [23]. Additional research is needed to understand the influence of income, employment type and status, employment-related mandates, insurance status, rurality, and education on the likelihood of accepting a COVID-19 vaccine when it becomes available [7, 9, 15, 16, 22, 23]. So far, there have been discussions on vaccine prioritization (e.g., for healthcare or frontline workers), but little has been done as it relates to the planning of vaccination for the most disadvantaged, vulnerable, and marginalized populations who may continue to remain at a disadvantage as was seen with COVID-19 outcomes in these populations (i.e., a greater number of deaths and severe infections). Cost and access-based planning on COVID-19 vaccines for such groups remain a major obligation for policymakers to ensure successful population-based and widespread immunization if and when the COVID-19 vaccines are available for the general population.

An interesting finding of this study that warrants further exploration is sex-based differences and vaccine hesitancy based on having children at home. In the final regression model, vaccine hesitancy odds were higher for females ( $44 \%$ higher than males) and those with children at home (34\% higher). Callaghan et al. and Fisher et al. found higher rates of COVID-19 vaccine refusal in women in the U.S. and Dror and colleagues found in an Israeli study that males were more likely to accept the potential COVID-19 vaccine and having children at home was a negative predictor for accepting vaccines. In contrast, in the global study by Lazarus and colleagues, study participants who were American males were more likely to have a positive response towards getting a COVID-19 vaccine compared to females in the U.S. Similarly, a May 2020 study by Malik and colleagues found that males (72\%) were more likely to accept COVID-19 vaccine than females $(63 \%)$ [7, 15-17, 23]. In general, women are more likely to practice preventive behaviors and avoid risk behaviors (e.g., influenza vaccination rates in the US and wearing of face masks to prevent COVID-19 infections) [24-26]. However, the results of COVID-19 vaccine hesitancy have been mixed. Additional research is needed on sex, parenthood, and family structure for COVID-19 vaccine hesitancy in light of influential factors such as risk perception, access to healthcare and health literacy, attitudes and beliefs, differential COVID-19 vulnerability and comorbidity burden based on sex, pre-existing vaccine hesitancy, safety and efficacy concerns, the effect of conspiracy theories associated with vaccines (e.g., for children and autism risk, deleterious effects of a vaccine potentially compromising the ability to care for children. unknown long-term sequelae, the seriousness of the infection due to vaccine), just to name a few [7, 15-17, 23-26].

The perceived threat of COVID-19 and political affiliation in the US stood out among the strong predictors of vaccine hesitancy in this study. These findings can be explained by the current polarized sociopolitical climate and individual COVID-19 risk perceptions across the US. COVID-19 has been highly politicized in the US. Throughout the pandemic, COVID-19 risk perception and mitigation have been discussed and explored via a variety of national polls and studies keeping in mind sociodemographic factors and political affiliation [17, 18, 24, 27]. For example, the wearing of face masks became a divisive issue and ended up becoming a "culture war" with a greater divide in compliance based on gender, age, and political affiliation [24, 27]. Even while the trials for the COVID-19 vaccine were going on worldwide, many Americans did not believe COVID-19 was a serious problem or the topmost concern in the country. Republicans, Democrats, and other participants in polls and studies differed in their opinion with certain groups less likely to believe in the seriousness of COVID-19, the efficacy of masks, scientific guidance on controlling the pandemic, and willingness to take a COVID-19 vaccine when it became available $[4,6,17,18,24,27]$. To reach the levels of reasonable herd immunity or for successful mass COVID-19 immunization, tailored and community-based interventions that can address the political divide and risk perception differences will be needed along with the plans to distribute the COVID-19 vaccines.

Scientific organizations, public health experts, and media outlets are beginning to educate the general population about the COVID-19 vaccine to increase accurate information and to decrease vaccine hesitancy. Several key recommendations are offered for communicating information about COVID19 vaccines with the general population, and in particular, populations having high vaccine hesitancy. Communication strategies and techniques to be used during the ongoing 
pandemic should be transparent and honest, accurate and truthful, multimodal and frequent, inclusive and have partnerships with community members and healthcare professionals, able to acknowledge the uncertainty and quickly changing guidance, focusing on the engagement of official entities and scientific sources, avoiding partisan or motivated messages, create a sense of shared and bipartisan support for preventive behaviors and vaccines, able to use role models and respectable public figures, and combat misinformation, myths, misperceptions, and conspiracy theories $[4,6,8-10$, $12,21,23,27]$. After scientific validation, policymakers must develop and endorse policies and environmental support systems that foster the promotion of COVID-19 vaccination programs.

Several limitations may have affected the results of this study. The results are restricted by all threats to the validity and reliability inherent to survey study designs (e.g., reliance on self-reported behaviors, recall bias in participants, socially desirable responses, and the inability to establish cause-and-effect relationships). Moreover, there are many other characteristics of individuals (e.g., pre-existing vaccine hesitancy) that could have played a role in whether or not an individual reported vaccine hesitancy (e.g. previously had reactions and side effects from vaccines). Finally, a threat to the external validity is that the sample is limited in nature and extent (e.g., limited to those with computers or mobile phones and understanding of the online survey environment). Despite these limitations, our study on vaccine hesitancy is among the few and larger studies in the US. Also, the majority ( $>50 \%$ ) of our sample consisted of adult Americans who were Whites, females, non-Hispanic, married, employed full-time, urban or suburban residents, 26-60 years old, and with an annual household income less than $\$ 60,000$. These numbers closely resemble the US population distribution as per the Census and labor statistics making our study sample representative of the US population to a great extent $[13,14,16,23]$.

\section{Conclusions}

In this large community-based study on COVID-19 vaccine hesitancy in the U.S, it was found that almost a fifth (22\%) of the respondents were hesitant to take these vaccines if they are available. Differences in vaccine hesitancy were based on sociodemographic characteristics such as sex, race, ethnicity, education, income, employment status, and place of residence. Also, political affiliation and perceived COVID-19 threat were strong predictors of COVID-19 vaccine hesitancy. Various factors may be contributing to this vaccine hesitancy such as preexisting indecisiveness, historical mistrust with health care especially among minorities, cost-related concerns, and lower levels of awareness. Along with vaccine deployment and distribution efforts, additional research is needed to understand the complex interplay of a variety of individual and social characteristics that influence vaccine hesitancy to ensure broader coverage with COVID19 vaccines. Educational and policy-level interventions that are evidence-based must be implemented to address these issues and promote COVID-19 immunization programs. The rates of willingness to be vaccinated might change now given the availability of the vaccines, but frequent and untoward effects of vaccines may reduce those rates. Along with factors identified in this study, the long-term effects of the vaccines will influence the uptake of COVID-19 vaccines.

Funding This research received no external funding.

\section{Compliance with Ethical Standards}

Conflict of interest Authors have no conflicts of interests to declare.

\section{References}

1. Centers for Disease Control and Prevention. (2020). CDC COVID data tracker. Retrieved from https://covid.cdc.gov/covid-data-track er/\#cases_casesper100klast7days.

2. Cutler, D. M., \& Summers, L. H. (2020). The COVID-19 pandemic and the \$16 trillion virus. JAMA, 324(15), 1495-1496.

3. Cohen, J. (2020). Vaccine wagers on coronavirus surface protein pay off. Science, 370(6519), 894-895. https://doi.org/10.1126/ science.370.6519.894.

4. Branswell, H. (2020). The Covid-19 vaccines are a marvel of science. Here's how we can make the best use of them. Retrieved from https://www.statnews.com/2020/12/02/how-society-canmake-the-most-of-covid-19-vaccines/.

5. Centers for Disease Control and Prevention. (2020). How CDC is making COVID-19 vaccine recommendations. Retrieved from https://www.cdc.gov/coronavirus/2019-ncov/vaccines/recom mendations-process.html.

6. Ball, P. (2020). Anti-vaccine movement could undermine efforts to end coronavirus pandemic, researchers warn. Nature, 581(7808), 251. https://doi.org/10.1038/d41586-020-01423-4.

7. Dror, A. A., Eisenbach, N., Taiber, S., Morozov, N. G., Mizrachi, M., Zigron, A., et al. (2020). Vaccine hesitancy: The next challenge in the fight against COVID-19. European Journal of Epidemiology, 35(8), 775-779.

8. Badur, S., Ota, M., Öztürk, S., Adegbola, R., \& Dutta, A. (2020). Vaccine confidence: The keys to restoring trust. Human Vaccines \& Immunotherapeutics, 16(5), 1007-1017.

9. National Academies of Sciences, Engineering, and Medicine. (2020). Framework for equitable allocation of COVID-19 vaccine. Retrieved from https://www.nap.edu/catalog/25917/frame work-for-equitable-allocation-of-covid-19-vaccine.

10. McAteer, J., Yildirim, I., \& Chahroudi, A. (2020). The VACCINES Act: Deciphering vaccine hesitancy in the time of COVID19. Clinical Infectious Diseases, 71(15), 703-705.

11. World Health Organizations. (2019). Ten threats to global health in 2019. Retrieved from https://www.who.int/news-room/spotl ight/ten-threats-to-global-health-in-2019. 
12. Harrison, E. A., \& Wu, J. W. (2020). Vaccine confidence in the time of COVID-19. European Journal of Epidemiology, 35(4), 325-330.

13. Khubchandani, J., \& Price, J. H. (2020). Public perspectives on firearm sales in the United States during the COVID-19 pandemic. Journal of the American College of Emergency Physicians Open. https://doi.org/10.1002/emp2.12293.

14. Price, J. H., Dake, J. A., Murnan, J., Dimmig, J., \& Akpanudo, S. (2005). Power analysis in survey research: Importance and use for health educators. American Journal of Health Education, 36(4), 202-209.

15. Lazarus, J. V., Ratzan, S. C., Palayew, A., Gostin, L. O., Larson, H. J., Rabin, K., et al. (2020). A global survey of potential acceptance of a COVID-19 vaccine. Nature Medicine. https://doi. org/10.1038/s41591-020-1124-9.

16. Fisher, K. A., Bloomstone, S. J., Walder, J., Crawford, S., Fouayzi, H., \& Mazor, K. M. (2020). Attitudes toward a potential SARSCoV-2 vaccine: A survey of US adults. Annals of Internal Medicine. https://doi.org/10.7326/M20-3569.

17. Callaghan, T., Moghtaderi, A., Lueck, J. A., Hotez, P. J., Strych, U., Dor, A., et al. (2020). Correlates and disparities of COVID19 vaccine hesitancy. Available at SSRN 3667971. https://doi. org/10.2139/ssrn.3667971.

18. Kaiser Family Foundation. (2020). Poll: Most Americans worry political pressure will lead to premature approval of a COVID19 vaccine; half say they would not get a free vaccine approved before election day. Retrieved from https://www.kff.org/coron avirus-covid-19/press-release/poll-most-americans-worry-polit ical-pressure-will-lead-to-premature-approval-of-a-covid-19vaccine-half-say-they-would-not-get-a-free-vaccine-approvedbefore-election-day/.

19. Webb, F. J., Khubchandani, J., Striley, C. W., \& Cottler, L. B. (2019). Black-white differences in willingness to participate and perceptions about health research: Results from the PopulationBased HealthStreet Study. Journal of Immigrant and Minority Health, 21(2), 299-305.

20. Ferdinand, K. C., Nedunchezhian, S., \& Reddy, T. K. (2020). The COVID-19 and influenza "Twindemic": Barriers to influenza vaccination and potential acceptance of SARS-CoV2 Vaccination in African-Americans. Journal of the National Medical Association. https://doi.org/10.1016/j.jnma.2020.11.001.

21. Quinn, S. C., Jamison, A. M., \& Freimuth, V. (2020). Communicating effectively about emergency use authorization and vaccines in the COVID-19 pandemic. American Journal of Public Health. https://doi.org/10.2105/AJPH.2020.306036.

22. Shen, A. K., Hughes, R., IV, DeWald, E., Rosenbaum, S., Pisani, A., \& Orenstein, W. (2020). Ensuring equitable access to COVID19 vaccines in the US: Current system challenges and opportunities. Health Affairs. https://doi.org/10.1377/hlthaff.2020.01554.

23. Malik, A. A., et al. (2020). Determinants of COVID-19 vaccine acceptance in the US. EClinicalMedicine, 26, 100495. https://doi. org/10.1016/j.eclinm.2020.100495.

24. Khubchandani, J., Saiki, D., \& Kandiah, J. (2020). Masks, gloves, and the COVID-19 pandemic: Rapid assessment of public behaviors in the United States. Epidemiologia, 1(1), 16-22.

25. Griffith, D. M., et al. (2020). Men and COVID-19: A biopsychosocial approach to understanding sex differences in mortality and recommendations for practice and policy interventions. Preventing Chronic Disease, 17, E63.

26. Applewhite, A., Stancampiano, F. F., Harris, D. M., Manaois, A., Dimuna, J., Glenn, J., et al. (2020). A retrospective analysis of gender-based difference in adherence to influenza vaccination during the 2018-2019 season. Journal of Primary Care \& Community Health. https://doi.org/10.1177/2150132720958532.

27. Bruine de Bruin, W., Saw, H. W., \& Goldman, D. P. (2020). Political polarization in US residents' COVID-19 risk perceptions, policy preferences, and protective behaviors. Journal of Risk and Uncertainty. https://doi.org/10.1007/s11166-020-09336-3.

Publisher's Note Springer Nature remains neutral with regard to jurisdictional claims in published maps and institutional affiliations. 